\title{
Response-independent outcome presentations weakens the instrumental response-outcome association.
}

Byron Crimmins ${ }^{1}$, Molly McNulty ${ }^{1}$, Vincent Laurent, Genevra Hart* \& Bernard W. Balleine* Decision Neuroscience Lab, School of Psychology, UNSW Sydney

1. Equal contribution

* Corresponding authors

Running Head: Contingency degradation and devaluation

\section{Correspondence:}

Genevra Hart, Bernard Balleine

Decision Neuroscience Lab

School of Psychology

UNSW SYDNEY

Botany Road, Randwick, NSW 2052, Australia

email: bernard.balleine@unsw.edu.au

tel: +61435659949

\section{Footnote to first page:}

The research reported in this manuscript was supported by grants from the Australian Research Council, DP200103401 and DP160105070, and by a Senior Investigator Grant from the NHMRC of Australia, GNT1175420, to Bernard Balleine. The authors thank John Bolzan for his help in designing and constructing the vertical lever used in Experiment 3 and Tony Dickinson for helpful discussions throughout this project. 


\begin{abstract}
The present paper explored the fate of previously formed response-outcome associations when the relation between $\mathrm{R}$ and $\mathrm{O}$ was disrupted by arranging for $\mathrm{O}$ to occur independently of R. In each of three experiments response independent outcome delivery selectively reduced the $\mathrm{R}$ earning that $\mathrm{O}$. Nevertheless, in Experiments 1 and 2, the R continued to show sensitivity to outcome devaluation, suggesting that the strength of the R-O association was undiminished by this treatment. These experiments used a two-lever, two-outcome design introducing the possibility that devaluation reflected the influence of specific Pavlovian leveroutcome associations. In an attempt to nullify these incidental Pavlovian cues, Experiment 3 used a single bidirectional vertical lever that rats could press left or right for different outcomes. Again, response-independent outcome presentation selectively depressed the performance of the $\mathrm{R}$ that delivered the response-independent $\mathrm{O}$. However, in this situation, the response independent $\mathrm{O}$ also reduced the sensitivity of $\mathrm{R}$ to outcome devaluation; whereas the non-degraded $\mathrm{R}$ was sensitive to devaluation, the degraded $\mathrm{R}$ was not. We conclude that selective degradation of the instrumental contingency can weaken a specific R-O association while leaving other R-O associations intact. Furthermore, the use of a bidirectional vertical lever in Experiment 3 revealed that instrumental manipulanda, such as levers or chains, produce Pavlovian cues capable of forming incidental associations with the instrumental outcome, obscuring the relative influence of R-O associations after various manipulations.
\end{abstract}

\title{
Keywords
}

Instrumental conditioning; Pavlovian conditioning; context conditioning; bidirectional control; outcome devaluation; contingency degradation. 


\section{Introduction}

There is now considerable evidence that the acquisition of instrumental conditioning is determined by the formation of an association between the instrumental response $(R)^{1}$ and its specific consequences or outcome $(\mathrm{O})$. This evidence has come from a number of demonstrations using a variety of techniques. For example, using an outcome devaluation preparation, several labs have found (following Adams \& Dickinson, 1981) that hungry rats trained on two instrumental responses, for example to press a lever or pull a chain, for different outcomes, such as pellets and sucrose, reduce the performance of one response relative to the other after devaluation of its associated outcome (Balleine \& Dickinson, 1998; Colwill \& Rescorla, 1985). Similarly, studies assessing outcome specific Pavlovianinstrumental transfer have found that, after such two-response two-outcome training, a Pavlovian stimulus previously paired with one of the two outcomes selectively increases the performance of the instrumental response that earned that outcome (Colwill \& Rescorla, 1988; Corbit et al., 2001).

Nevertheless, although these results imply that highly specific R-O associations are formed during instrumental training, they rely for this conclusion on manipulations of the incentive value of the outcome rather than the R-O association itself. In fact, attempts to manipulate the $\mathrm{R}-\mathrm{O}$ association provide a third source of evidence for specific R-O learning. For example, following Hammond (1980), Balleine \& Dickinson (1998) gave hungry rats two-response two-outcome training with the outcomes delivered at a fixed probability in each second of the session in which its associated response was performed. After this training they attempted selectively to degrade one R-O association by continuing to deliver the outcomes contiguously in this way while also delivering one of the two outcomes independently of performance at the same probability but calculated in each second in which a response was not performed. As a consequence of this training, therefore, one outcome could only be earned by performing its associated response (i.e., the non-degraded response) whereas the other could be earned at the same rate whether its associated response was performed or not (i.e., the degraded response - see also Colwill \& Rescorla, 1986; Dickinson \& Mulatero, 1989). This degradation procedure has been found selectively to reduce performance of the degraded response both during the course of training and in a subsequent extinction test, and so has been argued to reveal that the rat is sensitive both to the causal status of its instrumental responses and the specificity of the R-O associations controlling their performance (see Balleine, 2019 for review).

Despite demonstrations that degrading the R-O contingency produces reliable and responsespecific decrements in instrumental performance, there is little direct evidence that it does so by reducing the strength of the R-O association. To our knowledge, Rescorla was the first directly to assess this claim. He (Rescorla, 1992a) trained rats on the two-response twooutcome procedure with the outcomes delivered on a variable interval schedule and then degraded the R-O relationship for one response by allowing rats to perform that response while delivering both outcomes randomly on a variable time schedule. To assess the effect of the unpaired outcomes, Rescorla used an outcome-specific transfer test for which distinct stimuli were paired with the two outcomes, with those stimuli then presented when the rats were again allowed to perform the two instrumental responses. Rescorla (1992a) found that, even in the face of extensive unpaired outcome presentations, the Pavlovian cues exerted a similar, response-selective, outcome-specific Pavlovian instrumental transfer effect on both

\footnotetext{
${ }^{1}$ We refer to instrumental actions in this paper as instrumental responses, abbreviated $\mathrm{R}$ instead of A, and, therefore, to the associative structure of instrumental conditioning as a response-outcome, or R-O, rather than an action-outcome, or A-O, association.
} 
the degraded and the non-degraded instrumental responses (see also Colwill, 2001).

Although this experiment suggests that R-O associations remain intact after degradation, there are several issues with this claim. First, the shift from a variable interval to a variable time schedule may have only weakly, if at all, affected the R-O association given evidence that, like extinction, such treatments modify performance via competing inhibitory connections (Bouton et al., 2016; Colwill, 1991; Rescorla, 1993b). Furthermore, despite claims to the contrary, outcome-specific transfer may not in fact provide an adequate test of the strength of R-O associations. A number of analyses of transfer effects have been developed in recent years suggesting that, rather than providing evidence for selective R-O associations, such tests reflect the impact of O-R associations on performance (Balleine \& Ostlund, 2007; see Cartoni et al., 2016, for review). Consistent with this claim, several studies have reported that outcome-specific transfer is unaffected by outcome devaluation (Balleine \& Ostlund, 2007; Holland, 2004; Rescorla, 1994) and, indeed, when O-R and R-O associations are put into conflict, outcome devaluation affects performance based on the R-O association (Ostlund \& Balleine, 2007; Rescorla, 1992b).

Given these potential sources of weakness in the evidence, it remains possible that, rather than leaving the R-O association intact, contingency degradation affects performance by reducing the strength of the R-O association. Based on the evidence described above, therefore, the current series of experiments sought to re-evaluate this claim, assessing the effect of response independent outcome presentations on the R-O association using sensitivity to outcome devaluation as the metric for associative strength rather than transfer.

\section{Experiment 1: Effect of contingency degradation on outcome devaluation}

The design of Experiment 1 is shown in Figure 1. We began this assessment using a standard within-subjects instrumental conditioning protocol in which hungry rats were trained on two different lever press responses (left or right lever; R1 and R2) with each earning a distinct outcome (pellets or sucrose solution; $\mathrm{O} 1$ and $\mathrm{O} 2$ ) delivered on a probabilistic schedule of reinforcement. We then sought to degrade one R-O relationship, as previously described (Balleine \& Dickinson, 1998), by giving the rats sessions in which the outcomes continued to be earned as in training but with one of the two outcomes also delivered unpaired with lever pressing at the same probability.

\begin{tabular}{l|c|c|c|c} 
Training & Degradation & Retraining & Deval test & Deval test (ctxt B) \\
\hline R1-O1 & R1-O1/O1 & R1-O3 & O1: R1 vs. R2 & O1: R1 vs. R2 \\
R2-O2 & R2-O2/O1 & R2-O3 & O2: R1 vs. R2 & O2: R1 vs. R2
\end{tabular}

Figure 1. Design of Experiment 1. Two responses (R1 and R2; left lever press, right lever press) were each trained with a distinct food outcome (O1 and $\mathrm{O} 2$, grain pellets and sucrose solution). All rats then underwent contingency degradation during which one of the previously earned outcomes was also delivered independently of responding (R1-O1/O1, R2-O2/O1). They were retrained with each response earning a common outcome (O3, polycose), after which R1 and R2 were tested for their sensitivity to outcome devaluation induced by specific satiety on $\mathrm{O} 1$ and $\mathrm{O} 2$ on separate days (order counterbalanced). Each rat was, therefore, tested with each outcome devalued and non-devalued across the two tests. A second devaluation test was conducted after additional retraining in a context that differed from the training context (ctxt B). 
Degradation is expected to reduce responding of the degraded relative to the non-degraded response and, as our aim in this experiment was to compare the sensitivity of these responses to outcome devaluation, we first attempted to make performance on the levers more comparable by re-training them using a common polycose outcome. For the test we used outcome-specific satiety to devalue the outcomes, conducting two outcome devaluation extinction tests across successive days. For one test the outcome of the degraded response was devalued whereas for the other the outcome of the non-degraded response was devalued, in counterbalanced order. This allowed us to compare the effects of devaluation on both the degraded and non-degraded responses when their associated outcome was devalued and when it was not devalued.

If instrumental contingency degradation weakens the performance of a response because it weakens its specific R-O association then the performance of the degraded response should be insensitive to outcome devaluation, or at least significantly less sensitive than the nondegraded response. If, however, degradation does not affect the strength of the R-O association then both responses should be similarly sensitive to outcome devaluation.

\section{Methods}

Subjects. Subjects were experimentally naive, Long Evans rats $(\mathrm{n}=22,12$ males, 10 females), obtained from the UNSW breeding facility. Rats weighed 200-350g (females) and 300-450g (males) and were between 13 and 17 weeks old at the start of the experiment. All rats were housed in groups of 3 or 4 in a climate-controlled colony room on a $12 \mathrm{~h}$ light-dark cycle (lights on from 07:00-19:00). Four days prior to the start of training, rats were put on food restriction of 7-20g of home chow per rat per day, fed in the afternoon, and were weighed every 2 nd day to ensure they remained $\sim 90 \%$ of their initial feeding weight. They were handled daily. All experimental procedures were approved by the Animal Ethics Committee at UNSW Sydney.

Apparatus. All behavioral training and testing procedures were conducted in 16 operant chambers (MED Associates, Vermont), enclosed in light and sound attenuating shells. Each chamber contained a pellet dispenser, able to deliver a $45 \mathrm{mg}$ grain food pellet (Bioserve Biotechnologies), and a pump, which delivered $0.2 \mathrm{ml}$ of a $20 \%$ sucrose solution (white sugar diluted in water), into a recessed magazine. The chambers were illuminated during all behavioral sessions with a $3 \mathrm{~W}, 24 \mathrm{~V}$ house light located on the wall opposite to the magazine. The chamber contained two retractable levers that could be inserted independently on the left- and right-side of the magazine. Presses of the levers and head entries into the magazine were automatically detected and recorded. All behavioral training and recording of behavioral responses were controlled by two computers positioned in a separate room running the MED-PC software (MED Associates, Vermont). Outcome devaluation occurred in a separate room to behavioral training and was conducted in 16 transparent plastic tubs with a metal grate lid. Outcomes were presented in a dish (pellets) or a bottle (sucrose) with the room lights off during the pre-feeding session.

\section{Procedure}

Magazine training. Rats were given two sessions of magazine training of 20-30 minutes each. During the session, 20 grain pellets and 20 sucrose solution reinforcers were each delivered on independent 60 -sec schedules (i.e., roughly 1 outcome of each type each 60 secs) with the constraint that the two outcomes could not be delivered within $2 \mathrm{~s}$ of each other.

Lever training. Rats were trained to press the two levers on a random ratio (RR) schedule, one for the sucrose and the other for the grain pellets, counterbalanced across subjects; i.e., for 
half the left lever delivered sucrose and the right lever pellets with the remainder receiving the opposite arrangement. Each rat was given two lever training sessions per day: one on each response-outcome arrangement. All rats were initially trained on a continuous reinforcement schedule (CRF, where every response is reinforced) with the session terminating when they earned 30 outcomes or after 60 minutes. This criterion was repeated on CRF sessions for each rat until it had been reached (which was achieved within 1-2 sessions for all rats). Subsequently, rats were trained on a RR 5 schedule (in which every 5 th response was reinforced on average) for 3 days, a RR10 schedule for the next 3 days, and finally a RR20 schedule for the last 3 days of training. Outcomes were delivered probabilistically calculated in each second in which there was a response; i.e., the probability of an outcome given a response $(\mathrm{p}(\mathrm{O} \mid \mathrm{R})$ was $0.2,0.1$ and 0.05 for RR5, RR10 and RR20 schedules, respectively.

Contingency degradation training. Following the final session of RR20 lever training, rats underwent 6 days of contingency degradation, consisting of two 20-minute sessions per day, spaced at least 90 minutes apart. In these sessions, lever presses continued to earn either pellets or sucrose on the RR20 schedule as during training, however, in addition to any earned outcomes, one of the outcomes was also delivered in an unpaired arrangement at the same probability but in each second in which a response was not performed (i.e., probability of an outcome given no response $(\mathrm{p}(\mathrm{O} \mid \mathrm{noR})=0.05)$. Unpaired outcomes were delivered with the constraint that only one outcome could be delivered in any 1-second period and could not be delivered within 1-second of a lever press. Importantly, the same unpaired outcome was delivered during both sessions meaning that, in one session the unpaired outcome was the same as that earned on the lever whereas in the other session it was different from that earned on the lever. Half of the rats received pellets as the unpaired outcome and half the sucrose solution. The order of degraded and non-degraded training sessions was alternated across days for each animal.

Lever press retraining. After contingency degradation, rats were given retraining on both levers for a common polycose outcome ( $20 \%$ Poly-Joule in water) to ensure performance on the degraded and nondegraded levers was comparable before the test phase. Rats were first preexposed to the polycose one hour per day in their home cage for 3 days to reduce neophobia. They were then re-trained across 6 days on the two lever press responses in separate sessions. Each session lasted until 30 outcomes were earned or 60 minutes had passed. The first day of training was on an RR5 schedule and the following 5 days on an RR10 schedule.

Outcome devaluation and choice test. For two days prior to outcome devaluation, rats were pre-exposed to the outcome devaluation chambers for one hour each day without any outcomes present, again to reduce neophobia. Rats were then given an outcome devaluation test over 2 days. Outcome devaluation consisted of one hour of free access to either grain pellets or sucrose solution (in counterbalanced order across animals) in the outcome devaluation chamber. Immediately after feeding, rats were given a 5-minute choice extinction test in the operant chambers in which both levers were available but no outcomes were delivered. On the second day rats were tested after pre-feeding the other outcome.

Outcome devaluation test after a context switch. In the final phase of the experiment, rats were re-trained across 2 days on the same instrumental responses for polycose in the operant chambers with the context modified with a different wall decoration, black and white stripes instead of clear plexiglass, floor material, black plexiglass instead of a grid floor, and smell, a peppermint odor added to the bedding, to provide a new context (Context B). Rats then underwent the same devaluation pre-feeding procedure as previously described and were then given two choice extinction tests over the next 2 days in this new context as described above. 
Statistical Analysis. Training and retraining data were analysed using within-subjects orthogonal contrasts comparing the to-be-degraded and to-be-non-degraded levers controlling the per-contrast error rate at alpha $=0.05$ (Hays, 1973). Response rates across each day of contingency degradation were converted into baseline percentage scores by dividing the mean response rate on each lever by the mean of the same lever across the last 3 days of instrumental training, in order to account for pre-existing biases or preferences prior to degradation. These baseline scores were then assessed statistically in the same manner as initial training by comparing the degraded to the non-degraded lever and testing for a linear trend across degradation training.

Exclusions. Prior to assessing outcome devaluation after degradation, we applied a conservative criterion to exclude from the primary analyses any rats that failed to show contingency degradation. The criterion we applied was a simple numerical one: responding on the degraded lever more than the non-degraded lever by greater than one standard deviation on both the last day of degradation training and the first day of retraining. The magnitude of devaluation for the remaining rats was analysed using a 2 × 2 within-subjects factorial design with orthogonal contrasts testing for the main effect of devaluation, main effect of degradation, and the devaluation $\mathrm{x}$ degradation interaction, controlling the per-contrast error rate at alpha $=0.05$ (Hays, 1973).

\section{Results}

Training. The rates of performance on the to-be-degraded vs. the to-be-non-degraded response during instrumental training are presented in Figure 2 (left panel) and, as is clear from the figure, there were no differences in overall rate of responding at any stage (Fs $<1.0)$. This was maintained across the last three days of instrumental RR20 training (Fs $<1.0$ ), and these response rates were used as baseline measurements against which to assess changes in responding across contingency degradation training. There was a significant linear trend for the to-be-degraded and the to-be-non-degraded response across training days, indicating that rats significantly increased lever pressing (per minute) across days $(\mathrm{F}(1,21)=371.572, \mathrm{p}<$ $.00001)$. The was no significant difference in this linear trend across responses indicating that the increase in press rate did not differ on the two levers $(\mathrm{Fs}<2.0)$.
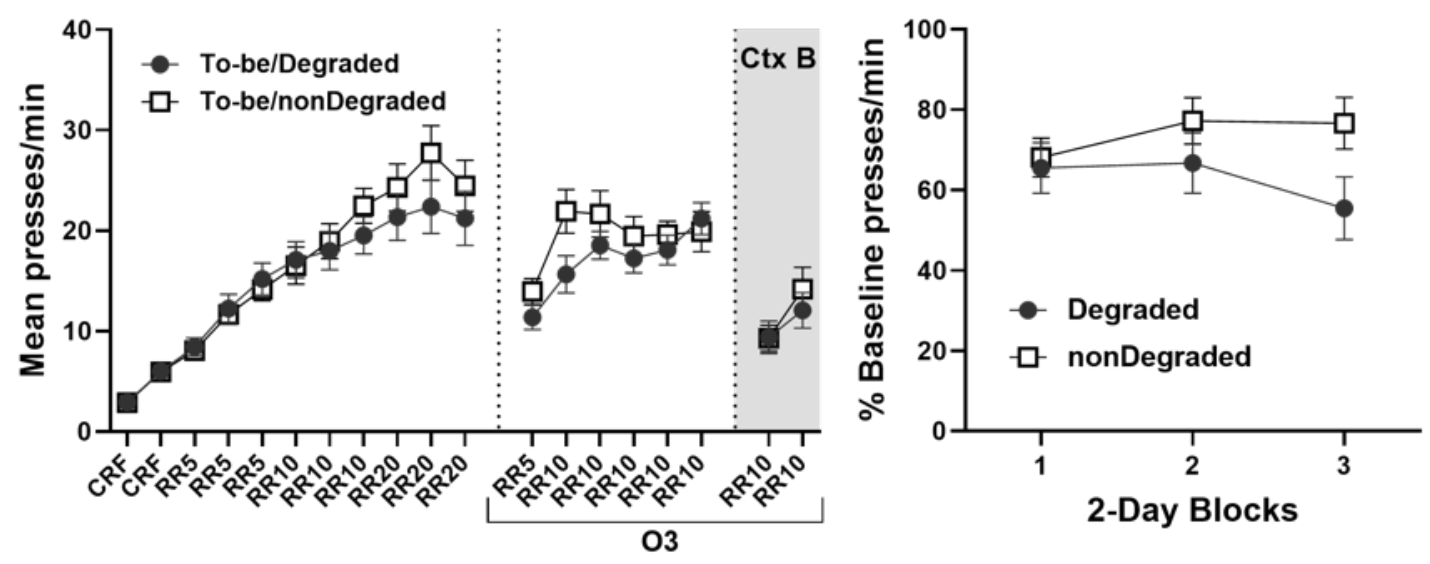

Figure 2. Instrumental training and contingency degradation in Experiment 1. Left panels show mean responses per minute on the to-be-degraded and to-be-nondegraded responses during initial training (left), during retraining on the common outcome (centre) and between devaluation tests in the new context, ctxt B (right). The right-hand panel shows the presses per minute on the degraded and nondegraded levers during contingency degradation training as a percentage of baseline, averaged over 2-day blocks. Error bars $= \pm 1$ SEM. 
Contingency Degradation. The effect of contingency degradation training is presented in the right panel of Figure 2, which shows a reduction in the degraded response relative to the nondegraded response developed over the course of training. Responding during contingency degradation was analysed according to a baseline correction, which equated for any preexisting preferences for one lever/outcome over the other. Baseline-corrected response rates on each lever during contingency degradation were expressed as the percentage of the average response rate on the same lever across the last 3 days of instrumental training. Averaged across all days of training, there were no differences in overall response rates $(\mathrm{F}(1,21)=2.8, \mathrm{p}$ $=0.1$, however a difference emerged across training, shown by a significant difference between the degraded and non-degraded levers across the last 2 days of training $(F(1,21)=$ $6.6, p=0.018)$, indicating that contingency degradation selectively reduced responding on the degraded relative to the non-degraded lever. Furthermore, although there was no significant linear trend for the degraded and non-degraded levers across contingency degradation $(\mathrm{F}(1,21)<1)$, there was a significant interaction between the linear trend for each lever and the overall responding on both levers $(\mathrm{F}(1,21)=7.8, \mathrm{p}=0.01)$, indicating that the difference between the degraded and the non-degraded responses increased across the course of the degradation training sessions.

Retraining. Rats were re-trained to press both levers for a common outcome, polycose in the same context as original training. This served to assess both any residual effect of contingency degradation and to equalize baseline response rates on the levers prior to the outcome devaluation test. The rates of responding during re-training with polycose are presented in Figure 2 (middle panel). Importantly, the difference between the degraded and non-degraded levers was maintained across the initial days of retraining; rats pressed the degraded lever significantly less than the non-degraded lever across days $1-2(F(1,21)=4.9, p$ $=.04$ ), indicating that contingency degradation training was effective in producing long- term suppression of the degraded lever. Responding on the two levers did not differ on Day 3 $(\mathrm{F}(1,21)=4.2, \mathrm{p}=0.05)$, or across the remaining 3 days of training $(\mathrm{Fs}<1.0)$. As such, retraining achieved the aim of making performance of the two responses comparable before the test phase.

Outcome devaluation test. We next assessed the effect of contingency degradation on the strength of the R-O association in the training context using an outcome devaluation test. In order to reliably assess this effect, we first assessed the magnitude of contingency degradation for each animal, and we separated those animals that failed to show any evidence of contingency degradation, and instead maintained a strong preference for the degraded lever, the criterion for which is described in the Statistical Analyses. There were 3 rats that met this criterion and that were excluded from the main analysis leaving $\mathrm{N}=19$.

The rate of responding on each lever across the 5-min choice test when it was valued and devalued ( 2 tests) for the remaining rats is presented in Figure 3. There was a significant main effect of outcome devaluation on responding, with rats responding more on the valued than the devalued lever $(\mathrm{F}(1,18)=11.4, \mathrm{p}=0.003)$, averaging across the degraded and nondegraded levers. Holding constant whether the lever was valued or devalued (i.e., averaging across valued and devalued levers), however, responding on the degraded or non-degraded lever did not differ significantly $(\mathrm{F}(1,18)=1.2)$. Furthermore, there was no significant interaction, indicating that the magnitude of the devaluation effect did not differ significantly for the degraded vs. the non-degraded lever $(\mathrm{F}(1,18)<1.0)$. Therefore, contingency degradation did not affect sensitivity to outcome devaluation when conducted in the same context as training. 
Outcome devaluation test after a context switch. One concern regarding the current design is the differential influence any context-outcome association might have on instrumental performance after outcome devaluation. If the context contributes to the vigor of responding then it is possible that, after devaluation of, say, $\mathrm{O} 1$, any context-O1 association could depress responding after devaluation of $\mathrm{O} 1$ relative to devaluation of $\mathrm{O} 2$, masking any effect of contingency degradation. To assess the effect of devaluation on each lever independently of such context effects, all rats were re-trained in a second context, Context B for two days with both responses earning the polycose (Figure 2 - right panel). There were no differences between rates of responding on the degraded and non-degraded lever across these two days, $\mathrm{F}(1,21)<1.0)$.

Rats were then given a second outcome devaluation test in Context B, presented in the right panel of Figure 3. Generally, the results looked similar to those of the first test. There was a significant main effect of outcome devaluation, with rats pressing more on the valued than the devalued lever $(\mathrm{F}(1,18)=7.7, \mathrm{p}=0.01)$, and no main effect of degradation, indicating response rates on the degraded and non-degraded levers did not differ, averaged across devalued and valued levers $(\mathrm{F}(1,18)<1.0)$. Again, although the effect looked smaller on the non-degraded than the degraded lever (the opposite of our prediction), the degradation $\mathrm{x}$ devaluation interaction was not significant, indicating the magnitude of the devaluation effect did not differ significantly for the degraded vs. the non-degraded lever $(\mathrm{F}(1,18)=1.4)$. Therefore, the test context appeared not to play a role in the effect of contingency degradation on the sensitivity of the instrumental response to outcome devaluation.
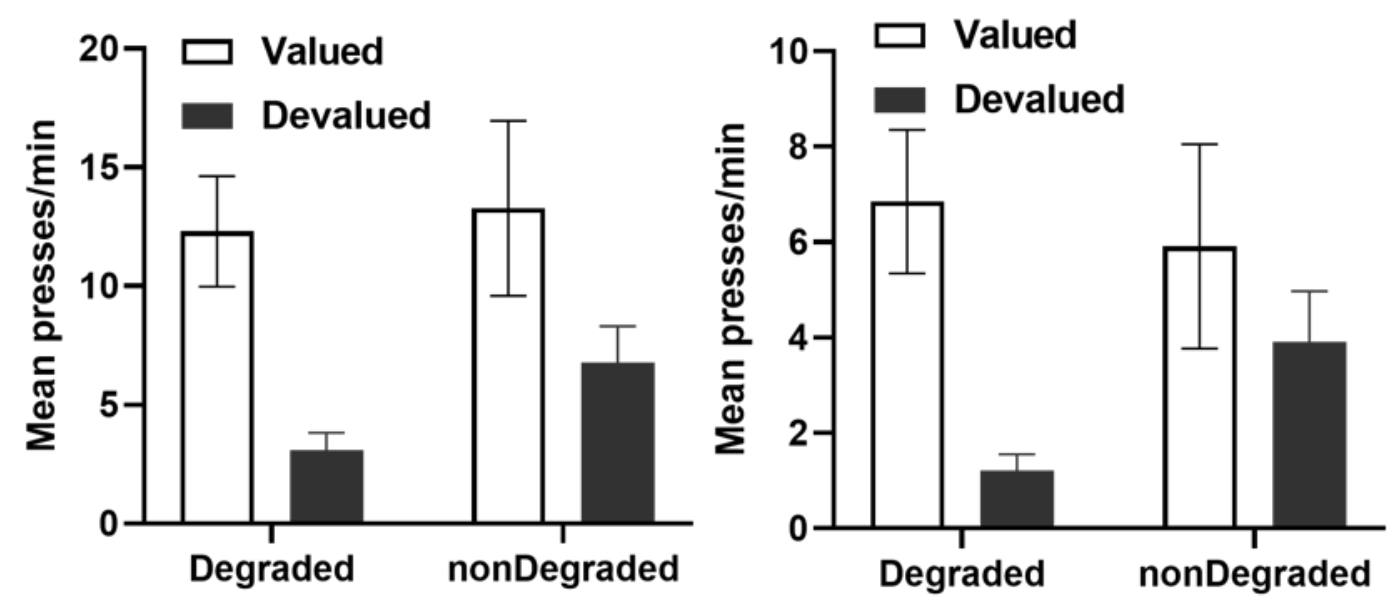

Figure 3. Devaluation tests after contingency degradation in Experiment 1. Left panel: Data from the first devaluation test presented separately as mean presses per minute on the degraded and nondegraded response when the outcome was devalued and when it was valued. Right Panel: Data from the second devaluation test conducted in context B separated for the degraded and nondegraded response when the associated outcome was devalued and when it was valued. Error bars $= \pm 1$ SEM.

\section{Discussion}

The aim of Experiment 1 was to determine if instrumental contingency degradation weakens the response-outcome association. We predicted that, if it did so, we should observe a reduced sensitivity to outcome devaluation leading to a reduced outcome devaluation effect on the degraded response. Prior to contingency degradation, the rats showed similar levels of responding on both levers, whereas, after contingency degradation, they responded significantly more on the non-degraded than the degraded lever, as expected. This suggests 
that the rats learned the selective R-O associations, and that their instrumental performance was sensitive to the change in contingency. This decreased responding on one lever compared to the other persisted over the first 3 days of the polycose retraining, further demonstrating the influence of degradation in these residual effects.

Nevertheless, contrary to our predictions, contingency degradation did not affect the sensitivity of the degraded response to outcome devaluation; there was no significant difference in the sensitivity of the degraded and the non-degraded levers in the outcome devaluation test. This was true even when we took steps to exclude those animals that did not show a numerical degradation effect or when we shifted the testing context in order to reduce any mediating effects of the context-outcome association on sensitivity to outcome devaluation. Given this pattern of results it seems reasonable to conclude, as Rescorla (1992a) concluded, that the strength of the underlying R-O association is not affected by responseindependent outcome presentations.

It is possible, however, that the context switch we used at test was simply insufficiently salient to reduce the influence of any differential context conditioning induced during contingency degradation training. To address this issue, we modified degradation training to avoid differential context conditioning across the two outcomes in Experiment 2.

\section{Experiment 2: The role of the experimental context.}

To address the potential influence of differential context conditioning in Experiment 1, Experiment 2 used a design similar to that developed by Rescorla (1992a - Experiment 1), shown in Figure 4. Rats were trained and tested using the same procedures as Experiment 1 except that we altered contingency degradation training so that both outcomes were presented unpaired with one of the lever press responses during the degradation sessions. In contrast, neither outcome was presented unpaired with the other lever press response during the nondegraded sessions. Although this treatment should be sufficient to degrade the contingency between one response and its training outcome relative to the other response, as both outcomes are presented unpaired during degradation, it should achieve this without the potential for the differential context conditioning we identified as a potential confounding influence in Experiment 1. If the association between the response independent outcome and the context was responsible for the continued sensitivity of the degraded response to outcome devaluation, then equating context conditioning for both outcomes should reduce the sensitivity of the degraded response to outcome devaluation relative to the non-degraded response.

\begin{tabular}{l|c|c|c} 
Training & Degradation & Retraining & Deval test \\
\hline R1-O1 & R1-O1/O1/O2 & R1-O3 & O1: R1 vs. R2 \\
R2-O2 & R2-O2 & R2-O3 & O2: R1 vs. R2
\end{tabular}

Figure 4. Design of Experiment 2. Rats were trained using the two response (R1 and R2) two outcome $(\mathrm{O} 1$ and $\mathrm{O} 2)$ procedure. They then underwent contingency degradation during which both $\mathrm{O} 1$ and $\mathrm{O} 2$ were also delivered independently of one response (R1) but not the other (R2) in different sessions. Rats were retrained using a common outcome (O3, polycose), after which $\mathrm{R} 1$ and $\mathrm{R} 2$ were tested for their sensitivity to outcome devaluation induced by specific satiety on $\mathrm{O} 1$ and $\mathrm{O} 2$ on separate days (order counterbalanced). 


\section{Methods and procedure}

Subjects. All procedures and statistical analyses were identical to Experiment 1 except that we used 24 rats (12 males, 12 females), and, as noted, made changes to contingency degradation training.

Degradation. Rats received the same amount of contingency degradation training as Experiment 1 and, in each degradation session, pressing the left or right lever continued to earn either a pellet or sucrose outcome (counterbalanced) at a fixed probability of $p=0.05$ (RR20). In this experiment, however, in one session both outcomes were also delivered unpaired with responding on one of the two levers at the same combined probability, i.e., $p=0.05$ ( $p=0.025$ for each outcome), with the constraint that only 1 outcome could be delivered in any 1-second period, and unpaired outcomes could not be delivered within 1second of a lever press. In the other session, the other lever press response was likewise reinforced on the same schedule (RR20), but no unpaired outcomes were delivered during that session. The R-O relationship that was degraded was counterbalanced between animals and the order of the degraded and non-degraded training sessions was alternated across days.

\section{Results}

Training. The mean press rate on each lever across each day of training is presented in Figure 5 (left panel). There were no differences between rates of responding on the to-bedegraded lever and to-be-non-degraded lever across any stages of instrumental training $(\mathrm{F}<$ 1.0). This was maintained across the last three days of instrumental RR20 training $(F<1.0)$, and these response rates were used as baseline measurements against which to assess changes in responding across contingency degradation training. There was a significant linear trend for the to-be-degraded lever and to-be-non-degraded lever across training days, indicating that rats significantly increased lever pressing (per minute) across days $(\mathrm{F}(1,23)=170.4, \mathrm{p}<$ $.00001)$, which did not interact with lever indicating that the increase in press rates did not differ across levers.
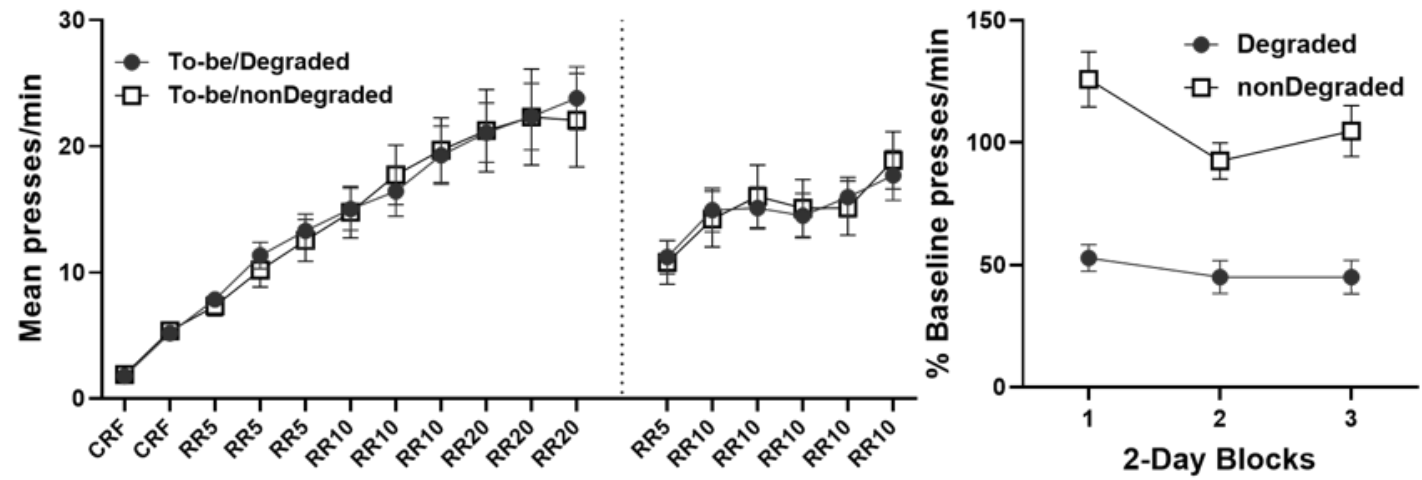

Figure 5. Training, retraining and contingency degradation data for Experiment 2. Left panel shows mean responses per minute on the to-be-degraded and to-be-nondegraded responses during initial training and during retraining for the polycose (centre). The right-hand panel shows the presses per minute on the degraded and nondegraded levers during contingency degradation training as a percentage of baseline averaged over 2-day blocks. Error bars $= \pm 1$ SEM.

Contingency Degradation. The effect of contingency degradation is shown in Figure 5 (right panel) and, as in Experiment 1, response independent outcome presentations clearly depressed performance of the degraded response. The press rates during contingency degradation were 
corrected for baseline and expressed as a percentage of the mean press rate on each lever for each rat across the last 3 days of instrumental training, averaged across all rats. A significant difference between responding on the degraded and non-degraded lever was observed throughout this training $(\mathrm{F}(1,23)=38.4, \mathrm{p}<.00001)$. Therefore, contingency degradation with both outcomes produced a significant and robust reduction in instrumental responding. There was no significant linear trend for the degraded and non-degraded levers across contingency degradation $(F(1,23)=3.6, p=0.07)$, nor a significant interaction between the linear trend for each lever and the overall responding on both levers $(\mathrm{F}(1,23)<1)$, indicating that the rate of linear change across sessions did not significantly differ between the degraded and the nondegraded levers.

Retraining. The mean rate of instrumental responding on the two levers across re-training with polycose is presented in Figure 5 (centre panel). Unlike Experiment 1, rats entered retraining with similar response rates on both levers; there were no differences between rates of responding on the previously degraded and non-degraded levers across day 1 of training $(\mathrm{F}<$ $1.0)$, nor averaged across the remaining days of retraining $(F s<1.0)$ and so no evidence of a residual effect of degradation.

Outcome Devaluation and Choice Test. A choice test in extinction conducted after outcome devaluation was used to assess whether the R-O association was weakened by degradation responding on the degraded vs. the non-degraded lever when the corresponding outcome was valued or devalued. As in Experiment 1, prior to the choice test we excluded rats that failed to show contingency degradation and maintained a strong preference for the degraded lever; 2 rats met the criterion for exclusion set in Experiment 1, leaving $\mathrm{N}=22$.

The mean presses per min on each lever across the 5-min choice test when the outcome was valued and devalued ( 2 tests) are presented in Figure 6. This figure reveals a modestly diminished devaluation effect on the degraded lever. Nevertheless, this was not borne out statistically. Averaging across the degraded and non-degraded levers, there was a significant main effect of outcome devaluation, with rats responding more on the valued than the devalued lever $(F(1,21)=9.5, p=0.006)$. In contrast there was no effect of degradation $(F(1$, $21)<1.0$ ) and no significant devaluation $x$ degradation interaction, indicating that the magnitude of the devaluation effect was not significantly different for the degraded vs. the non-degraded lever $(\mathrm{F}(1,21)<1.0)$.

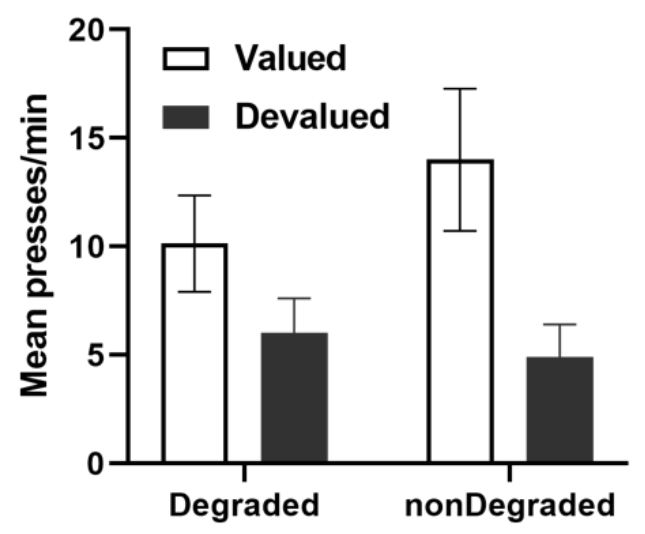

Figure 6. Devaluation test after contingency degradation in Experiment 2. Data from the devaluation test presented separately as mean presses per minute on the degraded and nondegraded response when the outcome was devalued and when it was valued. Error bars $= \pm 1$ SEM. 
Therefore, as observed in Experiment 1, Experiment 2 found no evidence that response independent outcome presentations weakened the instrumental R-O association, even when steps were taken to minimize the potentially confounding influence of differential context conditioning.

\section{Experiment 3: The role of Pavlovian stimuli.}

Of the factors known to modulate instrumental performance, among the best researched are the effects of Pavlovian stimuli. It is well established that when independently established Pavlovian predictors are presented on an instrumental baseline they can produce both general excitatory effects on performance and selective effects, depending on the outcome the stimuli and instrumental responses predict (Corbit \& Balleine, 2016). Thus, for example, Colwill and Rescorla (1988) demonstrated that a stimulus paired with, say, food pellets exerted a selective excitatory effect on responses that earn food pellets relative to responses earning a different outcome, say, a sucrose solution. It is possible, therefore, that a similar effect emerged in the current experiments, not due to stimuli trained elsewhere and presented during instrumental performance, but to the influence of incidental Pavlovian stimuli embedded in the instrumental conditioning situation from the beginning of training. In the current series of experiments, we have so far trained rats to press two levers to deliver the pellet and sucrose outcomes and, consequently, it is possible that the rats not only learned that the left and right lever press responses were associated with different outcomes, but that the left and right levers were too. If the latter associations provide a selective contribution to performance, then it is possible that the overall level of pressing on each lever reflects the contribution of the specific lever press-outcome associations $\left(\mathrm{R}_{\mathrm{LL}}-\mathrm{O} 1, \mathrm{R}_{\mathrm{RL}}-\mathrm{O} 2\right)$ plus the specific $\mathrm{S}-\mathrm{O}$ associations $\left(\mathrm{S}_{\mathrm{LL}}-\mathrm{O} 1, \mathrm{~S}_{\mathrm{RL}}-\mathrm{O} 2\right)$; i.e., on the left lever, net performance $(\mathrm{P})$ or $\mathrm{P}_{\mathrm{LL}}=\left(\mathrm{R}_{\mathrm{LL}}-\mathrm{O} 1\right)+\left(\mathrm{S}_{\mathrm{LL}}-\mathrm{O} 1\right)$ whereas, on the right lever, $\mathrm{P}_{\mathrm{RL}}=\left(\mathrm{R}_{\mathrm{RL}}-\mathrm{O} 2\right)+\left(\mathrm{S}_{\mathrm{RL}}-\mathrm{O} 2\right)^{2}$.

Along similar lines, although the reinforcer devaluation effect has been widely interpreted as evidence that instrumental conditioning is mediated by the R-O association, Colwill and Rescorla (1986) noted that the free operant procedure provides the opportunity for stimuli generated by the manipulanda to become selectively associated with the various outcomes via a Pavlovian process. Given the sensitivity of Pavlovian conditioning to reinforcer devaluation (Holland, 1979; Holland \& Rescorla, 1975), this analysis raises the possibility that the instrumental outcome devaluation effect is in fact mediated by the Pavlovian association between the manipulandum cues and the outcome rather than by that between the response and the outcome. Consequently, outcome devaluation could continue to affect instrumental performance after contingency degradation, not because the R-O association is intact but because devaluation selectively reduces the excitatory influence of Pavlovian stimuli on performance via an outcome-specific Pavlovian devaluation effect.

Colwill and Rescorla (1986), and subsequently Dickinson et al (1996), addressed this issue using a bidirectional vertical pole manipulandum that could be pushed to the left or right for distinct outcomes, thereby equating (i) the associations between the outcomes and the pole manipulandum and, therefore, (ii) any influence that pole-related stimuli produce on both responses. In these studies, instrumental outcome devaluation and contingency degradation effects continued to emerge, providing evidence for the influence of the R-O association on instrumental performance. Nevertheless, while confirming a role for R-O associations, these findings do not rule out the possibility that specific S-O associations can also influence

\footnotetext{
${ }^{2}$ Note that there is also likely to be a contribution to performance by S-R associations, a suggestion considered previously by various theories (Dickinson \& Balleine, 1993; Perez \& Dickinson, 2020); but, as this contribution shouldn't differ across levers or be affected by either degradation or devaluation, it is not considered further.
} 
instrumental performance. It remains possible, therefore, that such associations were at work in Experiments 1 and 2 and confounded our attempts to assess the influence of response independent outcomes on the strength of the R-O association.

To evaluate this claim we sought to remove any differential influence of Pavlovian manipulandum-outcome associations on instrumental performance using a bidirectional manipulandum (see Figure 7). This manipulandum was a retractable vertical lever, designed and constructed in the UNSW School of Psychology, that could be pushed left to earn one outcome and right to earn an alternative outcome. This vertical lever was able to be locked in one or both directions - thereby restricting the responses able to be performed during training - and unlocked during a choice extinction test.
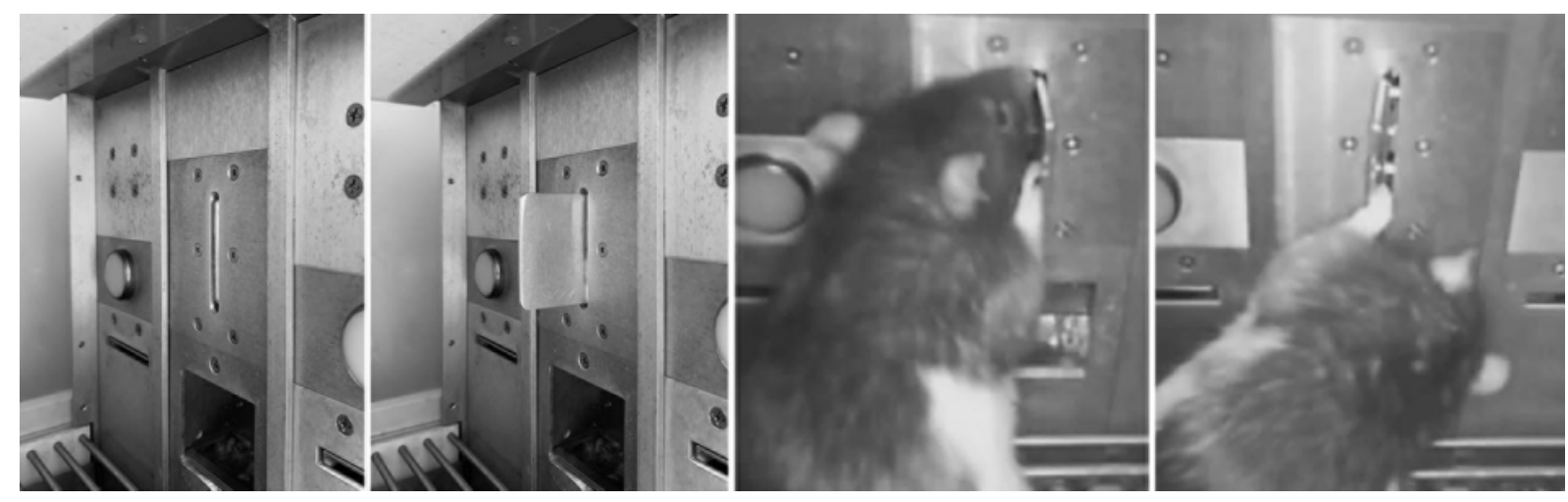

Figure 7. The bidirectional, vertical lever used in Experiment 3. Left panel: Shows the positioning of the lever in the operant chamber and its profile when retracted. Second left panel shows the profile of the lever when extended. Second-right panel shows a rat interacting with the lever when pressing it left. Right panel shows a rat interacting with the lever when pressing it right.

The design of Experiment 3 is presented in Figure 8. Using this manipulandum the current experiment first established that performance was sensitive to outcome devaluation and contingency degradation. After a period of retraining using a common polycose outcome, we subsequently used a second devaluation test to assess the effect of the latter on sensitivity to the former. If response independent outcome presentations weaken the R-O association but this effect was obscured in Experiments 1 and 2 by the differential influence of manipulandum-outcome associations, then, by nullifying this influence using a bidirectional manipulandum, Pavlovian associations should no longer differentially affect performance and the degraded response should no longer be sensitive to outcome devaluation. If, however, degradation has no effect on the strength of the R-O association then both degraded and nondegraded responses should remain equally sensitive to outcome devaluation.

\begin{tabular}{l|c|c|c|c|c} 
Training & Deval test & Degradation & Deg. Test & Retraining & Deval test2 \\
\hline R1-O1 & O1: R1 vs. R2 & R1-O1/O1 & R1 vs. R2 & R1-O3 & O1: R1 vs. R2 \\
R2-O2 & O2: R1 vs. R2 & R2-O2/O1 & R1 vs. R2 & R2-O3 & O2: R1 vs. R2
\end{tabular}

Figure 8. Design of Experiment 3. Rats were trained using the two response (R1 and R2) two outcome (O1 and $\mathrm{O} 2)$ procedure. They then underwent a devaluation test followed by a contingency degradation training and a degradation extinction test to evaluate their capacity to interact with the bidirectional lever in a goal-directed manner. Rats were retrained using a common outcome (O3, polycose), after which R1 and R2 were tested for their sensitivity to outcome devaluation induced by specific satiety on $\mathrm{O} 1$ and $\mathrm{O} 2$ on separate days (order counterbalanced). 


\section{Methods}

Subjects. The subjects were identical to those described in Experiment 1, except we used 26 rats (10 males, 16 females).

Apparatus. All behavioral training and testing procedures were conducted in 8 operant chambers (MED Associates, Vermont) with similar enclosures, magazine, outcome delivery and house light as those described in Experiment 1. In this experiment, however, the chambers also contained a retractable vertical lever that could be inserted in the centre of the wall immediately above the magazine. The device enables a rat to perform two distinct responses on a single manipulandum. A motorised retractable arm extends a vertical plate or lever through a slit opening. When the motorised arm is retracted, the vertical lever is not accessible. When the arm is extended, the vertical lever can be pressed laterally in either direction. Pressure sensors, attached to the vertical lever, enable presses to be recorded digitally. Adjustable guards allow the vertical plate to be locked in one or both directions, thereby restricting the responses available. Photographs of the lever and a rat pressing it are shown in Figure 7.

As described in Experiment 1, presses on the lever and head entries into the magazine were automatically detected and recorded and all behavioral training and recording of behavioral responses was controlled by a computer positioned in a separate room running the MED-PC software (MED Associates, Vermont). Outcome devaluation again occurred in a separate room to behavioral training and was conducted in 16 transparent plastic tubs with a metal grate lid with the outcomes presented in a dish (pellets) or a bottle (sucrose) with the room lights off during the pre-feeding session.

\section{Procedure}

Magazine training. Rats received identical magazine training to that described in Experiment 1 , except 15 of each reinforcer, rather than 20 , were delivered over the same period.

Vertical lever training. Rats were trained to press the single vertical lever in one direction for sucrose and in the other direction for grain pellets, with half the rats trained to press left for pellets and right for sucrose and the remainder to press right for pellets and left for sucrose. The outcomes were delivered on a RR schedule, as in Experiment 1. Rats underwent two lever training sessions per day, each at least 30 minutes apart. For each session, the vertical lever was locked so that it could only be pressed in a single direction. The direction available was reversed for the second session each day and the order of these sessions was counterbalanced. Rats were trained on a CRF schedule for 6 days, RR 5 for 3 days, and RR10 for 3 additional days with the outcomes delivered on the probabilistic schedules as described in Experiment 1. Sessions were terminated after rats earned 20 rewards or after 25 minutes.

Outcome devaluation and choice test. At the end of training the rats were given an initial outcome devaluation assessment to establish the extent of control of performance on the vertical lever by the R-O associations. Other than the use of the vertical lever, the outcome devaluation and choice tests were identical to those described in Experiment 1 . The vertical lever was not locked for the test and the rats were free to press it in either direction. Again, two tests were conducted, one with the pellets devalued and another with the sucrose devalued with both tests ending in a 5 min choice extinction test.

Reminder training. Rats underwent 2 days of lever training, as previously described, with presses on the vertical lever earning the appropriate outcome on a RR10 schedule.

Contingency degradation training. After retraining the rats received contingency 
degradation training exactly as described in Experiment 1 except for the use of the vertical lever. Again, both the contingent and noncontingent outcomes were delivered at $\mathrm{p}=0.05$ and, again, two sessions per day were conducted with each response trained with an unpaired outcome that was either the same or different from the outcome earned by pressing the vertical lever. Sessions were terminated after 25 minutes.

Contingency Degradation test. To confirm that the contingency degradation treatment was effective, the day after the final contingency degradation training session the rats were given a contingency degradation extinction test. Rats were place into the operant chambers, and, after $1 \mathrm{~min}$, the vertical lever was extended and presses in each direction were recorded, for the next 10 minutes. For this test the lever was unlocked so that the rats were free to press the lever in either direction.

Vertical lever press retraining. After contingency degradation, rats were given 3 days of retraining on the vertical lever except for a common polycose outcome. Each day the rats were given two sessions of training; one in which they could press the vertical lever left for a polycose outcome and one in which they could press right for the same polycose outcome. Outcomes were delivered on a RR5 schedule for the first day and then on a RR10 schedule for the subsequent 2 days. Sessions were terminated after 25 min or 20 reinforcers were delivered.

Outcome devaluation and choice test. At the end of retraining the rats were given a second outcome devaluation test to establish the effect of contingency degradation on sensitivity to outcome devaluation using the vertical lever. This assessment was identical to the first devaluation test described previously. The vertical lever was not locked during the test and the rats were free to press it in either direction. Again, two tests were conducted across two days, one with the pellets devalued and another with the sucrose devalued in counterbalanced order, with both tests ending in a 5 min choice extinction test.

\section{Results}

Vertical lever training. Mean lever press response rates on the vertical lever in each direction (to-be-degraded and to-be-nondegraded), across the 12 days of initial training are presented in Figure 9 (left panel). The two responses were acquired similarly and there was no significant difference between the mean response rates in each direction $(\mathrm{F}(1,25)<1.0)$. There was a significant linear trend across training $(F(1,25)=425.6 \mathrm{p}<0.0001)$, but was no linear trend $\mathrm{x}$ lever response direction interaction $(\mathrm{F}(1,25)<1.0)$.

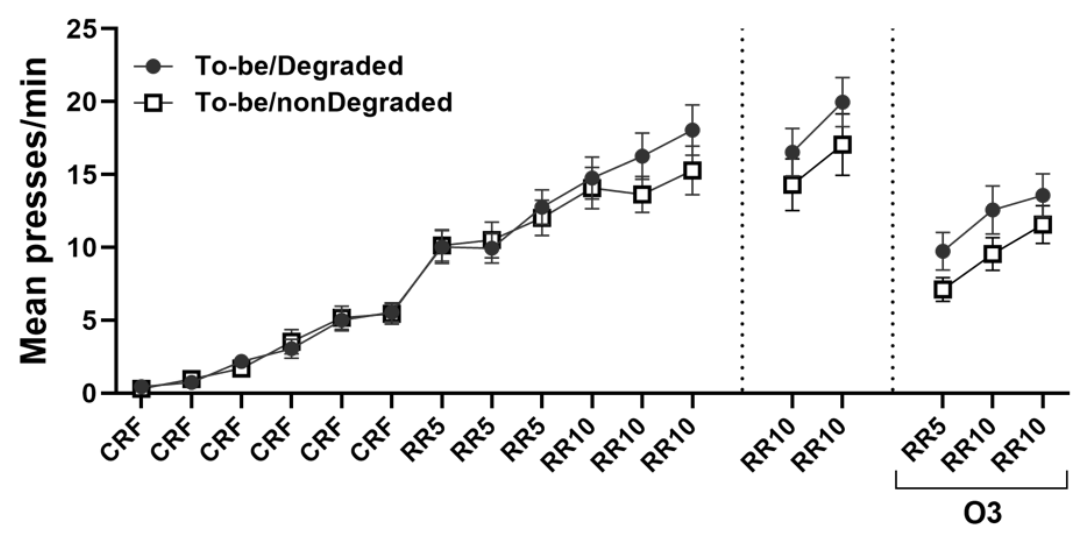

Figure 9. Instrumental training in Experiment 3. Panels shows the mean responses per minute on the to-be-degraded and to-be-nondegraded responses during initial instrumental training (left), during training after devaluation (centre) and during re-training after degradation on the common polycose outcome (right). Error bars $= \pm 1$ SEM. 
Outcome devaluation choice test. Rats were assessed for their sensitivity to outcome devaluation using the vertical lever manipulandum after initial training, which is presented in the left panel of Figure 10. A clear and similar outcome devaluation effect emerged on both the to-be degraded and to-be non-degraded response. There was a main effect of outcome devaluation $(\mathrm{F}(1,25)=28, \mathrm{p}<0.001)$, no main effect of lever direction (to-be-degraded vs tobe-nondegraded $)(\mathrm{F}(1,25)<1.0)$, and no interaction $(\mathrm{F}(1,25)<1.0)$. These data confirm that, at the end of instrumental training, performance on the bidirectional vertical lever was sensitive to outcome devaluation and so both the left and right push responses were demonstrably controlled by the R-O associations in both directions.
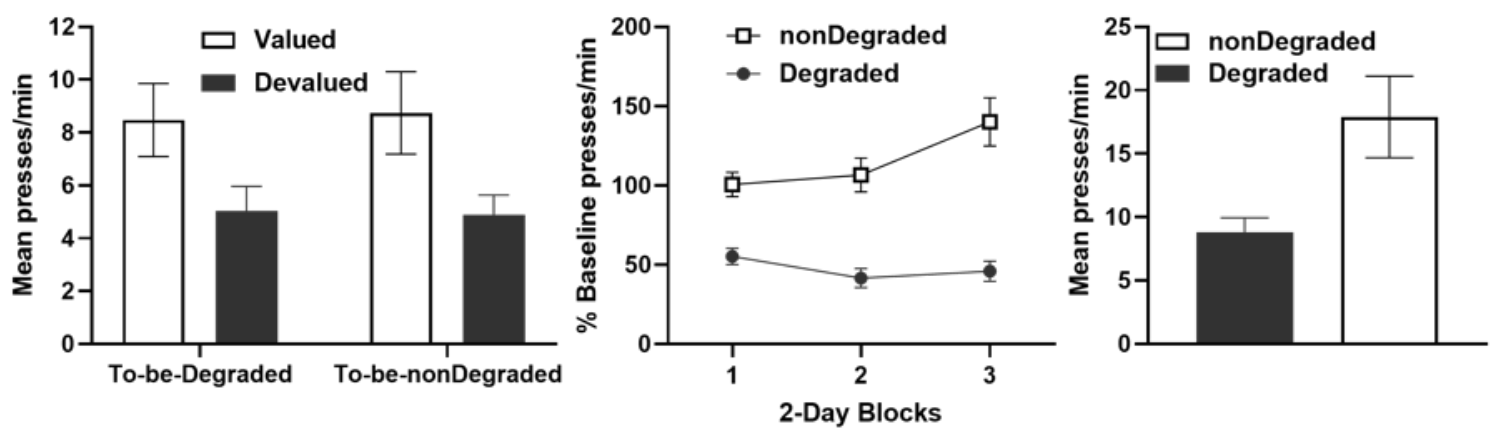

Figure 10. Outcome devaluation, contingency degradation and the degradation extinction test on the vertical lever in Experiment 3. Left panel shows the result of the first outcome devaluation test presented separately as mean presses per minute on the to-be-degraded and to-be-nondegraded responses when the outcome was devalued and when it was valued. Centre panel shows the effect of contingency degradation during training, whereas the right panel shows the effect of contingency degradation in a subsequent extinction test. Error bars $= \pm 1$ SEM.

Reminder training and Contingency degradation. Following the outcome devaluation tests, rats were given 2 days of reminder retraining on RR10 in the same manner as initial training (Figure 9 - centre panel). There were no differences in the rates of responding in either direction across these two days $(\mathrm{F}<1.0)$, and these response rates were then used as the baseline measurements against which to assess changes in responding across contingency degradation training.

Rats then underwent 6 days of contingency degradation training followed by an extinction test, as described. The mean percentage of baseline response rates on each direction (degraded and non-degraded) across training and test are presented in the center and right panels of Figure 10, respectively. There was a clear contingency degradation effect in the training data, with a significant main effect of degradation, rats responded less in the degraded than the nondegraded response direction, averaged across all training days $(F(1,25)=40.1 \mathrm{p}<0.001)$. The linear trend across training was not significant when averaged across the two responses $(\mathrm{F}(1,25)=2.9, \mathrm{p}=0.1)$; however there was a significant linear trend $\mathrm{x}$ response (degraded vs nondegraded) interaction $(F(1,25)=14.5, p=0.001)$, indicating that the magnitude of difference between the degraded and nondegraded levers increased as training progressed.

The impact of contingency degradation was also assessed in a choice extinction test, presented in the right panel of Figure 10. In this test the rats made significantly fewer degraded than non-degraded responses, as confirmed by a significant main effect of degradation $(\mathrm{F}(1,25)=7.3, \mathrm{p}=0.012)$.

Instrumental retraining and outcome devaluation choice test. In order to re-establish baseline response rates, rats underwent 3 days of retraining with the vertical lever for a 
common outcome, polycose, presented in Figure 9 (right panel). The difference between the degraded and non-degraded responses persisted across the first day of re-training $(\mathrm{F}(1,25)=5.13, \mathrm{p}=0.03)$, was marginal on the second day $(\mathrm{F}(1,25)=3.56, \mathrm{p}=0.07)$, and was non-significant on the third day of re-training $(\mathrm{F}(1,25)=2.5, \mathrm{p}=0.13)$.

Rats were then tested again for sensitivity to outcome devaluation. As in previous experiments, we first excluded rats that showed a strong preference for the degraded response, using the criterion set in Experiment 1; i.e., rats were excluded if they showed greater responding on the degraded than the non-degraded response by $>1$ SD across the last day of contingency degradation training and in the contingency degradation extinction test. There were 3 rats that met this criterion and were excluded, leaving $N=23$. Responding in the devaluation test on the degraded and nondegraded responses when they were valued and devalued for the remaining rats is presented in Figure 11. A clear devaluation effect emerged but only on the non-degraded response. Unlike prior experiments, the devaluation effect was very clearly abolished on the degraded response. There was a significant main effect of devaluation $(\mathrm{F}(1,22)=8.3, \mathrm{p}=0.009)$ and no main effect of degradation $(\mathrm{F}(1,22)=2.2, \mathrm{p}=0.15)$. Critically, there was a significant devaluation $x$ degradation interaction $(F(1,22)=5.2, p=0.03)$, indicating that the magnitude of the devaluation effect on instrumental performance was greater on the non-degraded response than the degraded response. Simple effects analysis found a significant devaluation effect on the non-degraded $(F(1,22)=4.84, p=0.04)$ but not the degraded $(\mathrm{F}<1)$ response.

The results of Experiment 3 provide, therefore, the first direct evidence that response independent outcome presentations not only reduce instrumental performance they also weaken the R-O association, establishing that the strength of the R-O association can be increased and decremented in a bidirectional manner.

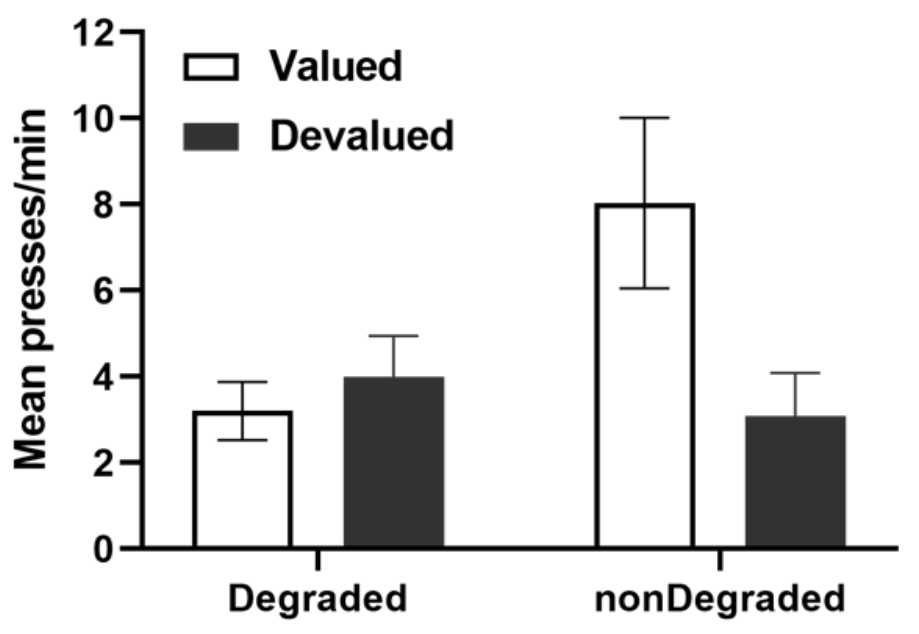

Figure 11. Devaluation test after contingency degradation in Experiment 3. The result of the outcome devaluation test on the vertical lever after contingency degradation presented separately as mean presses per minute on the degraded and nondegraded response when the outcome was devalued and when it was valued. Error bars $= \pm 1$ SEM. 


\section{General Discussion.}

In contrast to influential reports to the contrary, most notably that of Rescorla (1992a), the current study provides evidence that delivering the instrumental outcome independently of its previously associated instrumental response is sufficient to weaken the response-outcome association. Furthermore, this study also established the conditions under which such effects can be observed; that is, in a situation in which any influence of differential manipulandumoutcome associations on performance after outcome devaluation is nullified using a bidirectional manipulandum. Taken together, these experiments provide evidence that incidental Pavlovian stimuli in the instrumental situation, can influence instrumental performance and obscure the effect of treatments designed to influence the strength of the R$\mathrm{O}$ association.

The evidence for these claims comes from considering the results of Experiments 1 and 2 in the context of the results of Experiment 3 . In the former experiments, rats were trained on a two response two outcome procedure to press distinct left and right levers for different pellet and sucrose outcomes, after which degradation of one of the two R-O contingencies was induced by presenting the $\mathrm{O}$ independently of its associated $\mathrm{R}$. Nevertheless, despite a selective reduction in the performance of the degraded vs. the non-degraded response, the sensitivity of these responses to outcome devaluation appeared to be similar. It is possible, however, that delivering one outcome independently of the responses caused that outcome to become more strongly associated with the experimental context than the other outcome. If this is so, then perhaps devaluing that outcome reduced the contribution of the context to the vigor of the degraded response, reducing performance even though the R-O association was no longer intact. However, neither a shift in the test context in Experiment 1, nor our attempt to ensure that the instrumental outcomes were equally associated with the context during contingency degradation in Experiment 2, were effective in reducing sensitivity of the degraded response to outcome devaluation.

The other potential modulator of instrumental performance, and that which ultimately proved to be the source of our difficulties, is the influence of Pavlovian associations between the instrumental manipulanda and outcomes. If such associations provide a source of incentive motivation contributing to the vigor of instrumental performance, then perhaps removing that source through outcome devaluation was sufficient to produce the appearance that a specific $\mathrm{R}-\mathrm{O}$ association was intact when it was not. To assess this claim we developed a retractable bidirectional manipulandum, a vertical lever, that could be pushed left or right to deliver the pellet and sucrose outcomes. Although previous experiments using bidirectional manipulanda have reported evidence that instrumental performance remains sensitive to outcome devaluation (Colwill \& Rescorla, 1986; Dickinson et al, 1996), these demonstrations merely served to show, against various two process theories (Rescorla \& Solomon, 1968; Overmier $\&$ Trapold, 1972), that the influence of Pavlovian associations on performance is not the sole source of instrumental outcome devaluation effects. They do not and cannot demonstrate whether, and to what degree, incidental stimulus-outcome associations contribute to that performance. And, indeed, the results of Experiment 3 very clearly showed that nullifying the influence of differential manipulanda-outcome associations using a bidirectional manipulandum abolished the sensitivity of the degraded response, but not the non-degraded response, to outcome devaluation. Thus, only when steps were taken to remove differential Pavlovian influences on instrumental performance were we able to observe the deleterious effect of response-independent outcome presentations on the strength of the instrumental R-O association.

Although the current results do not agree with the conclusions of Rescorla's (1992a) 
assessment of the effects of response independent outcome delivery, his studies on this issue used Pavlovian instrumental transfer rather than outcome devaluation to assess the effect of this degradation treatment on the value of the R-O association. For reasons mentioned earlier, with hindsight this may not have been the most appropriate assay with which to assess this effect, given later findings that such methods may rely more on O-R than R-O relations. For example, the failure of transfer effects to be influenced by outcome devaluation (Rescorla, 1994; Holland 2004) and their opposing influence on performance across the course of training - with devaluation more influential earlier and Pavlovian cues more influential later in training (Holland, 2004) - are both particularly troubling in this regard. Furthermore, the increasingly large number of double dissociations between devaluation and transfer effects behaviorally (Corbit \& Balleine, 2003), pharmacologically (Laurent et al., 2012) and neurally (Corbit, Muir \& Balleine, 2001; Shiflett \& Balleine, 2010; see Balleine, 2019; Corbit \& Balleine, 2015 for reviews) make suggestions that both effects are mediated by, and can be used to assess, a common underlying associative structure particularly fraught. It may be argued that the account developed in the current paper has similar issues; that such findings make arguing Pavlovian processes can obscure changes in the R-O association equally problematic. However, in this case, the Pavlovian associations between the lever and outcome are present throughout instrumental training, rather than being trained elsewhere and added to the instrumental baseline, and are particularly likely to contribute to the vigor of performance because of their close relationship to the response itself over and above any effect of their association with a common $\mathrm{O}$.

In other studies, however, Rescorla did use outcome devaluation to assess the effect of various interference treatments on the R-O association. In one series, for example, he assessed the effect of order of training prior to devaluation and found following R1-O1 training by R2$\mathrm{O} 2$ did not affect the size of the outcome devaluation effect, whether O1 or O2 was devalued (Rescorla, 1993a). In another series he examined the effect of retraining the same response with a second outcome (e.g., R1-O1, followed by R1-O3) again finding that the initial R-O association survived this later training (Rescorla, 1996 - Experiment 1). Similarly, after training R1-O1 and R2-O2, outcome identity reversal to R1-O2, R2-O1 resulted in both R's being equally susceptible to the devaluation of its first- or second- earned outcome (Rescorla, 1996 -Experiments 2 and 3). In these cases, the strength of the R-O association appeared to be fully intact after retraining with a different outcome, suggesting that an instrumental $\mathrm{R}$ can maintain associations with several O's without any decrement in associative strength (see also Rescorla, 1995). Perhaps the most surprising such finding, however, is the case of extinction; i.e., the case in which the outcome of an $\mathrm{R}$ is changed from, say, $\mathrm{O} 1$ to nothing. In this case $\mathrm{R} 1-\mathrm{O} 1$ and R2-O2 training followed by R1- and R2- extinction was found to have no effect on sensitivity to outcome devaluation when compared to the effect on two other responses (R3-O1 and R4-O2) that were not extinguished (Rescorla, 1993a). For Rescorla, however, these extinction treatments were just less effective forms of response independent outcome delivery, which he argued might be superior for the study of extinction and that might be especially likely to yield associative loss (Rescorla \& Skucy, 1969). Although the current series maintained contiguous outcome delivery during the non-contingent sessions, there are good reasons to suppose that randomly presented response independent outcomes also produce decrements in instrumental behavior by affecting the R-O association. For example, Boakes and Halliday (1975) found no evidence of spontaneous recovery across sessions after a shift from a VI to a VT schedule, a finding that we have also observed using the degradation procedure described here (e.g., Balleine \& Dickinson, 1998).

Generally, therefore, although Rescorla (1992a) concluded that the associations established during instrumental training are remarkably resistant to the effects of various decremental 
operations, that "an R-Ol association apparently remains intact when $\mathrm{O} 1$ is replaced by another outcome, removed altogether, or made random with regard to R" (p.111), the findings of the current study raise a common issue with these results and with this interpretation, suggesting that incidental Pavlovian manipulanda-outcome associations may have contributed to the instrumental outcome devaluation effects observed in each of these cases. Reassessing these manipulations using a bidirectional manipulandum such as that used here will, therefore, provide an important next step in establishing the veracity of these claims.

A final implication of the current results worth raising is for theories of the R-O learning process underlying the acquisition of goal-directed instrumental responses. The dominant theoretical account of the formation of the R-O association advanced in the past was that based on associative theory, supposing that error signals generated as a result of the predicted consequences, or outcome, of a response increase the strength of association between the response and that outcome (Mackintosh \& Dickinson, 1979). As a consequence, on this view, the probability of the outcome given a response directly determines the strength of the response-outcome association, a claim that many areas of the literature have regarded as relatively uncontroversial (Maia, 2009; Solway \& Botvinick, 2012). More recently, however, this suggestion has been challenged, using as a catalyst the finding that rate of performance and sensitivity to outcome devaluation is greater on ratio than interval schedules of reinforcement even when the probability of an outcome given a response is matched on the two schedules (Dickinson et al., 1983). This challenge has generated an alternative account contending that the strength of the R-O association is determined, not by the probability of an outcome given the response, but by the strength of the correlation between the rate of responding and of outcome delivery across time; the rate-correlation theory (Perez \& Dickinson, 2020). Not only does this account explain the difference in sensitivity between ratio and interval schedules - the former generating a higher response-outcome correlation than the latter - it also anticipates the findings of the current experiments; whereas increases in the correlation between response and outcome rate increment the R-O association, decreases in that correlation decrement it; and response-independent outcome presentations turn out to be a particularly potent way to decrement that correlation. In the absence of outcome delivery, however, this account assumes that changes in the correlation cannot be calculated and, therefore, that the strength of the R-O cannot be changed; an assumption that predicts, in accord with Rescorla's various findings described above, that instrumental extinction will not affect the R-O association. Given this claim, the re-assessment of the effects of extinction on the strength of the R-O association may have implications for ratecorrelational theory too.

To the extent that Rescorla adopted any theoretical position regarding the R-O association, he appeared to favor a version of the associative model analogous to that he contributed to developing for Pavlovian conditioning (Rescorla, 1987; Rescorla \& Wagner, 1972). It is not surprising, therefore, that his experimental work in this area innovated on the approach he developed to study Pavlovian processes. Indeed, his work on instrumental conditioning along these lines was amongst his most creative, incorporating multiple discriminative stimuli, responses and outcomes in experimental designs of extraordinary power and incision to expose and manipulate selected features of the associative structure. Furthermore, some of the best evidence for this general view came from his manipulation of competing predictors, whether to establish the role of the discriminative stimuli in the hierarchical control of specific response-outcome associations (Rescorla, 1991) or, in the current context, in showing that the effect of response independent outcomes on performance can be blocked by signalling those outcomes (Colwill \& Rescorla, 1986). Such findings were somewhat at odds with the conclusion that response independent outcomes have no effect on the strength of the 
$\mathrm{R}-\mathrm{O}$ association and suggested to him that some other process was influencing performance, perhaps some form of inhibitory S-R association (Rescorla, 1993b). If the current results challenge the need for such a process, they nevertheless can also be understood as amplifying the claims of an associative view of instrumental conditioning. 


\section{References}

Adams, C. D., \& Dickinson, A. (1981). Instrumental Responding following Reinforcer Devaluation. The Quarterly Journal of Experimental Psychology Section B, 33(2b), 109-121. https://doi.org/10.1080/14640748108400816

Balleine, B. W. (2019). The Meaning of Behavior: Discriminating Reflex and Volition in the Brain. Neuron, 104(1), 47-62. https://doi.org/10.1016/j.neuron.2019.09.024

Balleine, B. W., \& Dickinson, A. (1998). Goal-directed instrumental action: Contingency and incentive learning and their cortical substrates. Neuropharmacology, 37(4-5), 407-419.

Balleine, B. W., \& Ostlund, S. B. (2007). Still at the choice-point: Action selection and initiation in instrumental conditioning. Annals of the New York Academy of Sciences, 1104, 147-171. https://doi.org/10.1196/annals.1390.006

Boakes, R. A., \& Halliday, M. S. (1975). Disinhibition and spontaneous recovery of response decrements produced by free reinforcement in rats. Journal of Comparative and Physiological Psychology, 88(1), 436-446. https://doi.org/10.1037/h0076216

Bouton, M. E., Trask, S., \& Carranza-Jasso, R. (2016). Learning to inhibit the response during instrumental (operant) extinction. Journal of Experimental Psychology: Animal Learning and Cognition, 42(3), 246-258. https://doi.org/10.1037/xan0000102

Cartoni, E., Balleine, B., \& Baldassarre, G. (2016). Appetitive Pavlovian-instrumental Transfer: A review. Neuroscience and Biobehavioral Reviews, 71, 829-848. https://doi.org/10.1016/j.neubiorev.2016.09.020

Colwill, R. M. (1991). Negative discriminative stimuli provide information about the identity of omitted response-contingent outcomes. Animal Learning \& Behavior, 19(4), 326336. https://doi.org/10.3758/BF03197893

Colwill, R. M. (2001). The effect of noncontingent outcomes on extinction of the responseoutcome association. Animal Learning \& Behavior, 29(2), 153-164. https://doi.org/10.3758/BF03192824

Colwill, R. M., \& Rescorla, R. A. (1985). Postconditioning devaluation of a reinforcer affects instrumental responding. Journal of Experimental Psychology: Animal Behavior Processes, 11(1), 120-132. https://doi.org/10.1037/0097-7403.11.1.120

Colwill, R. M., \& Rescorla, R. A. (1986). Associative Structures In Instrumental Learning. In Psychology of Learning and Motivation (Vol. 20, pp. 55-104). Elsevier. https://linkinghub.elsevier.com/retrieve/pii/S007974210860016X

Colwill, R. M., \& Rescorla, R. A. (1988). Associations between the discriminative stimulus and the reinforcer in instrumental learning. Journal of Experimental Psychology: Animal Behavior Processes, 14(2), 155-164. https://doi.org/10.1037/00977403.14.2.155

Corbit, L. H., \& Balleine, B. W. (2003). Instrumental and Pavlovian incentive processes have dissociable effects on components of a heterogeneous instrumental chain. Journal of Experimental Psychology. Animal Behavior Processes, 29(2), 99-106.

Corbit, L. H., \& Balleine, B. W. (2016). Learning and Motivational Processes Contributing to Pavlovian-Instrumental Transfer and Their Neural Bases: Dopamine and Beyond. Current Topics in Behavioral Neurosciences, 27, 259-289. https://doi.org/10.1007/7854_2015_388

Corbit, L. H., Muir, J. L., \& Balleine, B. W. (2001). The role of the nucleus accumbens in instrumental conditioning: Evidence of a functional dissociation between accumbens core and shell. The Journal of Neuroscience: The Official Journal of the Society for Neuroscience, 21(9), 3251-3260.

Dickinson, A., \& Balleine, B. W. (1993). Actions and responses: The dual psychology of behaviour. In Spatial Representation (pp. 277-293). Basil Blackwell. 
Dickinson, A., Campos, J., Varga, Z. I., \& Balleine, B. (1996). Bidirectional instrumental conditioning. The Quarterly Journal of Experimental Psychology. B, Comparative and Physiological Psychology, 49(4), 289-306. https://doi.org/10.1080/713932637

Dickinson, A., \& Mulatero, C. W. (1989). Reinforcer specificity of the suppression of instrumental performance on a non-contingent schedule. Behavioural Processes, $19(1-$ 3), 167-180. https://doi.org/10.1016/0376-6357(89)90039-9

Dickinson, A., Nicholas, D. J., \& Adams, C. D. (1983). The Effect of the Instrumental Training Contingency on Susceptibility to Reinforcer Devaluation. The Quarterly Journal of Experimental Psychology Section B, 35(1b), 35-51. https://doi.org/10.1080/14640748308400912

Hammond, L. J. (1980). The effect of contingency upon the appetitive conditioning of freeoperant behavior. Journal of the Experimental Analysis of Behavior, 34(3), 297-304.

Hays, W. L. (1973). Statistics for the social sciences (2d ed). Holt, Rinehart and Winston.

Holland, P. C. (1979). Differential effects of omission contingencies on various components of Pavlovian appetitive conditioned responding in rats. Journal of Experimental Psychology: Animal Behavior Processes, 5(2), 178-193. https://doi.org/10.1037/00977403.5.2.178

Holland, P. C. (2004). Relations Between Pavlovian-Instrumental Transfer and Reinforcer Devaluation. Journal of Experimental Psychology: Animal Behavior Processes, 30(2), 104-117. https://doi.org/10.1037/0097-7403.30.2.104

Holland, P. C., \& Rescorla, R. A. (1975). The effect of two ways of devaluing the unconditioned stimulus after first- and second-order appetitive conditioning. Journal of Experimental Psychology: Animal Behavior Processes, 1(4), 355-363. https://doi.org/10.1037/0097-7403.1.4.355

Laurent, V., Leung, B., Maidment, N., \& Balleine, B. W. (2012). $\mu$ - and $\delta$-opioid-related processes in the accumbens core and shell differentially mediate the influence of reward-guided and stimulus-guided decisions on choice. The Journal of Neuroscience: The Official Journal of the Society for Neuroscience, 32(5), 1875-1883. https://doi.org/10.1523/JNEUROSCI.4688-11.2012

Mackintosh, N. J., \& Dickinson, A. (1979). Instrumental type II conditioning. In Mechanisms of learning and motivation: A memorial volume to Jerzy Konorski (pp. 143-170). Erlbaum.

Maia, T. V. (2009). Reinforcement learning, conditioning, and the brain: Successes and challenges. Cognitive, Affective, \& Behavioral Neuroscience, 9(4), 343-364. https://doi.org/10.3758/CABN.9.4.343

Ostlund, S. B., \& Balleine, B. W. (2007). Selective reinstatement of instrumental performance depends on the discriminative stimulus properties of the mediating outcome. Animal Learning \& Behavior, 35(1), 43-52. https://doi.org/10.3758/BF03196073

Perez, O. D., \& Dickinson, A. (2020). A theory of actions and habits: The interaction of rate correlation and contiguity systems in free-operant behavior. Psychological Review, 127(6), 945-971. https://doi.org/10.1037/rev0000201

Rescorla, R. A. (1987). A Pavlovian analysis of goal-directed behavior. American Psychologist, 42(2), 119-129. https://doi.org/10.1037/0003-066X.42.2.119

Rescorla, R. A. (1991). Associative relations in instrumental learning: The Eighteenth Bartlett Memorial Lecture. The Quarterly Journal of Experimental Psychology Section B, 43, 123.

Rescorla, R. A. (1992a). Response-independent outcome presentation can leave instrumental R-O associations intact. Animal Learning \& Behavior, 20(2), 104-111. https://doi.org/10.3758/BF03200407 
Rescorla, R. A. (1992b). Response-outcome versus outcome-response associations in instrumental learning. Animal Learning \& Behavior, 20(3), 223-232. https://doi.org/10.3758/BF03213376

Rescorla, R. A. (1993a). Preservation of response-outcome associations through extinction. Animal Learning \& Behavior, 21(3), 238-245. https://doi.org/10.3758/BF03197988

Rescorla, R. A. (1993b). Inhibitory associations between S and R in extinction. Animal Learning \& Behavior, 21(4), 327-336. https://doi.org/10.3758/BF03197998

Rescorla, R. A. (1994). Transfer of instrumental control mediated by a devalued outcome. Animal Learning \& Behavior, 22(1), 27-33. https://doi.org/10.3758/BF03199953

Rescorla, R. A. (1995). Full preservation of a response-outcome association through training with a second outcome. Quarterly Journal of Experimental Psychology Section B., 48, 252-261.

Rescorla, R. A. (1996). Response-outcome associations remain functional through interference treatments. Animal Learning \& Behavior, 24(4), 450-458. https://doi.org/10.3758/BF03199016

Rescorla, R. A., \& Skucy, J. C. (1969). Effect of response-independent reinforcers during extinction. Journal of Comparative and Physiological Psychology, 67(3), 381-389. https://doi.org/10.1037/h0026793

Rescorla, R. A., \& Wagner, A. R. (1972). A theory of Pavlovian conditioning: Variations in the effectiveness of reinforcement and non reinforcement. In In AH. Black \& W.F. Prokasy (eds.), Classical conditioning II: current research and theory (pp. 64-99) (pp. 64-99). Appleton-Century-Crofts.

Shiflett, M. W., \& Balleine, B. W. (2010). At the limbic-motor interface: Disconnection of basolateral amygdala from nucleus accumbens core and shell reveals dissociable components of incentive motivation. The European Journal of Neuroscience, 32(10), 1735-1743. https://doi.org/10.1111/j.1460-9568.2010.07439.x

Solway, A., \& Botvinick, M. M. (2012). Goal-directed decision making as probabilistic inference: A computational framework and potential neural correlates. Psychological Review, 119(1), 120-154. https://doi.org/10.1037/a0026435 